\title{
The Bioactivity of Plasma Factors in Focal Segmental Glomerulosclerosis
}

\author{
Joanna Marszal Moin A. Saleem \\ Academic and Children's Renal Unit, University of Bristol, Southmead Hospital, Bristol, UK
}

\section{Key Words}

Podocyte - Focal segmental glomerulosclerosis •

Plasma factors · Nephrin · Podocin · Plasma exchange

\begin{abstract}
Focal segmental glomerulosclerosis (FSGS) is a devastating form of nephrotic syndrome, often leading to endstage renal failure after the failure of a succession of highly toxic therapies. It has long been thought to be caused by a circulating factor(s) that may be produced by cells of the immune system. Much research has focused on identifying such factor(s), including the development of a promising in vitro assay, which estimates glomerular permeability based on the swelling of isolated glomeruli in response to patients' plasma. This assay has also been used as the basis of testing plasma fractions for permeability activity, with no specific factor yet identified. Other studies have attempted to replicate proteinuria in whole animals, by injecting plasma or plasma fractions from focal segmental glomerulosclerosis patients, with inconsistent results. More recently there has been evidence that there may be either inhibitory or missing factor(s) in plasma, with respect to permeability. An additional major biological advance is a growing appreciation of the podocyte as the target cell in this disease, and an understanding of the key molecules involved. Putting together this knowledge, with the latest technological advances in protein identification, provides promising avenues towards finally solving the basis of this enigmatic disease.
\end{abstract}

Copyright $@ 2006$ S. Karger AG, Basel

KARGER
Fax +41613061234
E-Mail karger@karger.c

E-Mail karger@ka
www.karger.com

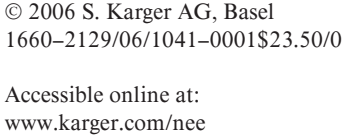

\section{Introduction}

Focal segmental glomerulosclerosis (FSGS) is a progressive form of idiopathic nephrotic syndrome, frequently leading to end-stage renal failure and, significantly, recurring in $30-60 \%$ of transplanted kidneys. Evidence for a circulating pathogenic 'factor' is therefore strong, though research into identifying such a factor has been frustrating. Recent insights suggest the possibility of a loss of critical proteins in FSGS. Applying these ideas in conjunction with an understanding of the biology of the likely target cell, the podocyte, will lead to a comprehensive picture of the pathogenesis of this disease.

\section{Background on Podocytes}

The glomerular podocyte is a highly differentiated epithelial cell. Among its main functions are contributing to the high pressure of capillaries, thereby counteracting the forces acting to distend them. Also, the arrangement and structure of podocytes determine the permeability properties of the glomerular filter. As yet few of the details surrounding the role of podocytes have been clarified, though it is widely accepted that damage to podocytes is a key factor in progressive renal disease. Podocyte injury results in proteinuria, a sign common to most glomerular disease. As a result, there is much interest in gaining an understanding of how the podocyte maintains its phenotype, and particularly with respect to nephrotic syndromes, in factors that might result in its injury.

Dr. Moin A Saleem, FRCP, PhD

University of Bristol

Children's Renal Unit, Bristol Royal Hospital for Children

Bristol BS2 8BJ (UK)

Tel. +44 117923 0000, Fax +44 117959 5438, E-Mail m.saleem@bristol.ac.uk 


\section{Structure and Development}

In the developing kidney, mesenchymal stem cells give rise to podocyte precursor cells that undergo a change in phenotype from immature 'cobblestone' cells to mature, highly differentiated cells, by responding to as yet unknown signals. Podocytes cover the urinary surface of the glomerular capillaries. Their cell bodies emit extensive primary processes that attach to the glomerular basement membrane with smaller foot processes.

The unique shape of the podocytes with their farreaching processes is maintained by a cytoskeleton, consisting of microtubules, intermediate filaments and microfilaments. The foot processes have a high density of microfilaments and especially of actin filaments, forming a complex structure capable of contraction [1].

Filtration slits are formed by the interdigitation of adjacent foot processes and are bridged by slit diaphragms (SDs). The integrity of the SD is maintained by a number of molecules expressed at this site. These include ZO-1, nephrin, podocin, CD2AP, FAT and P-cadherin. Nephrin, podocin and CD2AP link to the actin cytoskeleton, and mutations in any of those proteins cause massive congenital proteinuria [2]. Elucidating the role of SD proteins in maintaining podocyte integrity, and linking this to potential plasma factors, is an area of podocyte biology yet to be explored.

\section{Focal Segmental Glomerulosclerosis}

FSGS is the most common progressive glomerular disease in children and accounts for up to a quarter of all cases of idiopathic nephrotic syndrome in adults. Epidemiological studies have reported a dramatic increase in the incidence of FSGS, the most recent showing an increase of FSGS as a proportion of primary glomerulopathy from 17 to 59\% between 1992 and 2002 [3]. A fascinating new development is the report that activating mutations in the ion channel TRPC6 cause a familial form of FSGS [4, 5], providing the potential to link SD proteins to intracellular calcium flux and therefore multiple biological pathways.

Expression of SD proteins is altered in various glomerular diseases. In some studies of FSGS, nephrin expression is reduced or redistributed intracytoplasmically, though others do not support this $[6,7]$. A study by Patrakka et al. [8] found no evidence of such changes in patients with a number of conditions including FSGS, but suggested that the methods they used (in situ hybridiza- tion and immunohistochemistry) may not have been sensitive enough to detect small changes in nephrin expression. In toxin-induced models, nephrin is redistributed from a linear arrangement into intracellular granules [9].

Due to the clinical significance of FSGS, and the fact that it is regarded as a prototypical disease with regard to the circulation factor hypothesis, much research work has focused on identifying such a factor.

\section{Circulating Factor Hypothesis}

It has been suggested that FSGS may be caused by a circulating factor. Robert Shalhoub [10] was the first to put the hypothesis forward in 1974 and postulated that an abnormality of T-cell function results in secretion of a chemical mediator that damages the glomerular basement membrane.

The circulating factor hypothesis has since been the subject of much research, and the existence of an 'FSGS factor' appears to be supported by a number of important observations. Firstly, $30-40 \%$ of patients who receive a kidney transplant to treat their idiopathic FSGS experience recurrence of the condition, with this percentage increasing to $60-100 \%$ in patients receiving a second transplant after the first was lost to recurrent disease [11]. Secondly, immunosuppressive drug therapy and plasma exchange have proven to be effective in treating relapse in these patients.

Experimental analysis of the plasma removed during this therapy is of key importance in the work to try to identify this factor. Early work in this area involved animal models. Serum or plasma from FSGS patients injected into experimental animals was observed to cause proteinuria or albuminuria, though this is not always consistent [12]. Once FSGS patient plasma had been observed to have a definite effect on glomerular permeability, work began to characterize the circulating factor's responsibility.

In order to test for the presence of a circulating factor, an in vitro method for quantifying glomerular permeability in rats was utilized [13]. Glomeruli sieved from rats were used to measure the degree of capillary expansion produced by an oncotic gradient after incubation with various plasma solutions. The degree of capillary expansion was taken to be representative of the permeability to albumin. Plasma samples were used from normal patients and patients with idiopathic FSGS. Some of the FSGS patients had experienced recurrence of their condi- 
tion after kidney transplant, some had not. Incubation of the glomeruli with serum from normal patients resulted in no change in permeability to albumin. Patients with recurrence after transplant had a significantly higher mean value for albumin permeability than both the normal patients and the patients who had not experienced recurrence. Plasma samples taken from patients both before and after they received plasma exchange therapy demonstrated that plasmapheresis effectively reduces the glomerular permeability to albumin.

An important conclusion drawn from this work is that plasmapheresis removes a circulating factor responsible for increased glomerular permeability to albumin and that this factor is not rapidly resynthesized after removal.

Savin et al. [13] also performed some initial characterization of the factor they thought responsible. It was found to have an apparent molecular mass of approximately $50 \mathrm{kDa}$ and a weak anionic charge at $\mathrm{pH}$ 6.0. It was also soluble in $70 \%$ ammonium sulfate and bound to protein A.

Further work from this group to characterize the factor advanced with the development of an in vitro bioassay to measure its activity. The assay was used to quantify the activity of disease plasma fractions allowing a focus for purification efforts. The findings were in agreement with earlier work: the factor was soluble in $70 \%$ ammonium sulfate supernatant, and electrophoresis revealed a molecular weight of $30-50 \mathrm{kDa}$. This $70 \%$ supernatant induced proteinuria when injected into rats, whereas $70 \%$ supernatant from normal patients did not [14].

Recent work has provided more information on the character of the circulating factor and also strengthened the findings of Savin's work. Isolation of cellular proteins from glomeruli incubated with a fraction of FSGS plasma after isolation showed a decrease in the tyrosine phosphorylation of these proteins. Tyrosine phosphatase inhibition prevented the rise in albumin permeability that characterized the damaged podocytes. This suggested that the circulating factor might signal through altered phosphorylation of cellular proteins as part of its action [15].

However, these first steps towards characterizing the factor may not be as reliable as it was originally thought. The bioassays in use have been criticized for the reason that they are not advanced enough to take into account other endogenous permeability promoting substances (and their endogenous inhibitors) that may be present. The permeability factor (PF) activity of a plasma sample under analysis may vary greatly depending on these other substances regardless of the levels of 'the FSGS factor' [16]. It is also possible that samples are affecting glomerular endothelial cells in a manner that is not predictable, to cause capillary leak and swelling in vitro.

An important study into serum levels of PF activity demonstrated that levels do not actually change with relapse or remission of proteinuria as would be expected [17]. It was also shown that levels of PF activity remain unchanged with cyclosporin administration, despite the fact that this is an effective treatment. These findings are also at odds with the suggestion that the circulating factor is released by $\mathrm{T}$ cells, as cyclosporin is an immunosuppressant to which T cells are sensitive [17].

A possible explanation for these discrepancies is that the bioassays used to measure the PF activity were not sensitive enough to detect the tiny changes that may have been responsible for causing disease. It may also be that cyclosporin is effective because it acts locally on the glomerulus rather than suppressing all $\mathrm{T}$ cells, and in fact there is some recent evidence to support a direct protective effect on podocytes [18]. A third explanation is that $P F$ have differing activity in vivo and in vitro, lessening the relevance of the activity measured by the in vitro bioassay [16]. In any case, the circulating factor hypothesis has not been abandoned.

\section{Missing Factor Hypothesis}

An alternative and increasingly attractive perspective on FSGS pathogenesis is the hypothesis that rather than 'toxic' factor(s) being produced, there are either factors missing that are normally required for podocyte health or inhibitory factors to such proteins. The first study to suggest this documented that normal plasma prevents the induction of glomerular permeability by plasma from patients with FSGS recurrence [19]. A later study suggested the loss of endogenous PF inhibitors in the urine during proteinuria. Interestingly, when nephrotic urine was mixed with the nephrotic plasma from the same patient, PF activity of the plasma decreased. The same group looked at five patients with congenital NS, due to a podocin mutation, in whom it can be assumed that no pathogenic circulating factor is present. Plasma from these patients showed the same PF activity as idiopathic NS controls, and could be reduced to zero by adding homologous urine to the assay, but not urine from non-nephrotic controls [20]. This work must be viewed carefully; it may be that the loss of inhibitory factors in the urine only occurs 
Fig. 1. Is there an alteration of a dynamic balance that leads to foot process effacement and NS?

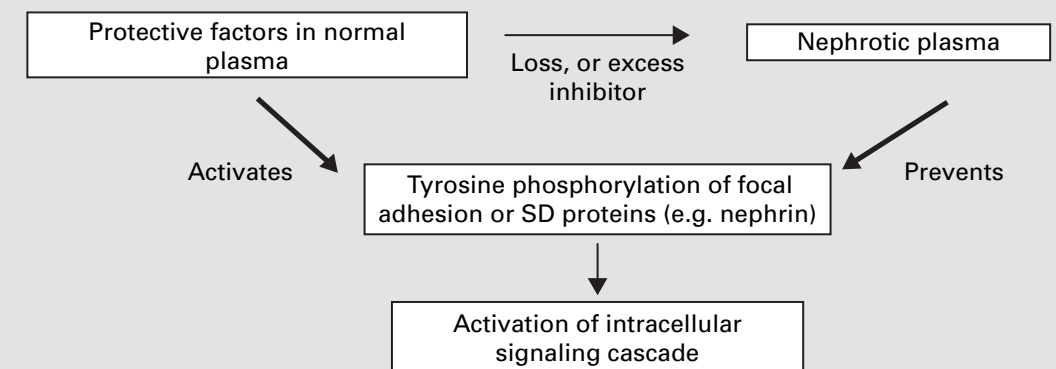

signaling cascade

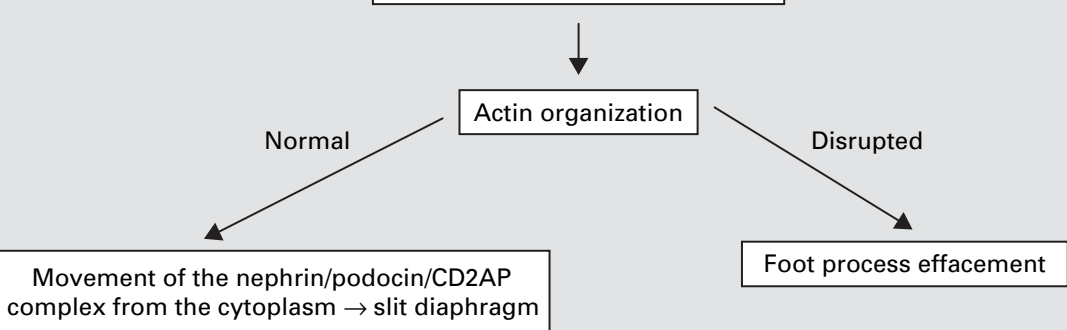

after the filtration barrier is damaged by another factor, and may not be the main cause of the disease process, but a perpetuating feature.

\section{Work Focusing on Podocytes}

Our own recent work in this context has utilized the generation of human conditionally immortalized podocytes that express key SD molecules when fully differentiated [21]. This has allowed, for the first time, a detailed molecular analysis of the effects of nephrotic plasma on podocyte SD proteins and cytoskeleton [22]. The data show that nephrin, podocin and CD2AP relocalize from the cell surface to the cytoplasm in response to relapse plasma, both from FSGS and non-FSGS nephrotic patients (supporting the hypothesis that nephrosis itself can perpetuate the disease). Intriguingly, incubating the cells in Fetal Calf Serum (FCS) also results in the same cytoplasmic relocation, which suggests that factor(s) in nor$\mathrm{mal} /$ remission human plasma are required for accurate positioning of the SD complex. Intracellular signaling was also stimulated by relapse plasma. Developing the theme further, we found that nephrin mutant podocytes are not able to relocalize their SD components or actin cytoskeleton in the same manner in response to normal human plasma, and display altered signaling in response to relapse and remission plasma. The signaling theme can be further developed now by consideration of TRPC6. We have found that normal human plasma suppresses (pathological) TRPC6 activity, and that nephrin is necessary for this suppression [23]. Overall, this indicates a role for the intact SD complex in regulating cytoskeletal patterning in the foot processes by mediating signaling events, and these events are triggered by factors in human plasma.

These data coincide with evidence that the nephrin/ podocin/CD2AP complex signals intracellularly, at least partly via nephrin tyrosine phosphorylation, though the ligand(s) are not yet known [24]. We already know that the mature podocyte is able to synthesize and secrete growth and PF of its own, for example vascular endothelial growth factor, which can act in an autocrine fashion on podocytes [25], and angiopoietin 1 [26], as well as cytokines (our own unpublished data). It therefore seems possible that the podocyte requires a milieu of crucial factors in its immediate environment to maintain its mature phenotype.

Thus a hypothesis can be proposed (fig. 1) of extracellular ligand(s) (at least some derived from circulating plasma), that signal to the SD complex to maintain the cytoskeletal and molecular integrity in podocyte foot processes in a dynamic manner. Some of these factors may be active or inhibitory in their ability to activate a signaling cascade, but the overall effect is to sustain a balance that preserves specialized podocyte function. 


\section{Future Directions}

The overwhelming likelihood is that FSGS is a heterogeneous condition, with the descriptive histology representing a final common pathway. Robust observations, and in particular the ability to reverse disease recurrence after plasma exchange and replacement with albumin solution, have intuitively pointed researchers towards a 'toxic' circulating factor. Recent evidence however, indicates a more complex explanation, with the possibility of inhibitory factor(s) also present. Our own view is that a variety of players are likely to be involved at different potencies in maintaining the mature podocyte phenotype, that this is disturbed in FSGS, and that plasma exchange somehow resets this delicate balance, when successful. Utilization of modern techniques, in particular application of proteomics methods to screen and identify plasma proteins that may be altered in FSGS, in combination with in vitro podocyte molecular changes as a readout of biological activity of these factors, is a highly promising avenue towards finally unraveling this difficult disease.

\section{References}

$>1$ Pavenstadt H, Kriz W, Kretzler M: Cell biology of the glomerular podocyte. Physiol Rev 2003;83:253-307.

$\checkmark 2$ Pollak MR: The genetic basis of FSGS and steroid-resistant nephrosis. Semin Nephrol 2003; 23:141-146.

>3 Dragovic D, Rosenstock JL, Wahl SJ, Panagopoulos $\mathrm{G}$, DeVita MV, Michelis MF: Increasing incidence of focal segmental glomerulosclerosis and an examination of demographic patterns. Clin Nephrol 2005;63:1-7.

-4 Winn MP, Conlon PJ, Lynn KL, Farrington MK, Creazzo T, Hawkins AF, Daskalakis N, Kwan SY, Ebersviller S, Burchette JL, PericakVance MA, Howell DN, Vance JM, Rosenberg PB: A mutation in the TRPC6 cation channel causes familial focal segmental glomerulosclerosis. Science 2005;308:1801-1804.

$\checkmark 5$ Reiser J, Polu KR, Moller CC, Kenlan P, Altintas MM, Wei C, Faul C, Herbert S, Villegas I, Avila-Casado C, McGee M, Sugimoto H, Brown D, Kalluri R, Mundel P, Smith PL, Clapham DE, Pollak MR: TRPC6 is a glomerular slit diaphragm-associated channel required for normal renal function. Nat Genet 2005;37:739-744.

6 Kim BK, Hong HK, Kim JH, Lee HS: Differential expression of nephrin in acquired human proteinuric diseases. Am J Kidney Dis 2002;40:964-973.

$>7$ Hingorani SR, Finn LS, Kowalewska J, McDonald RA, Eddy AA: Expression of nephrin in acquired forms of nephrotic syndrome in childhood. Pediatr Nephrol 2004;19:300-305.

-8 Patrakka J, Ruotsalainen V, Ketola I, Holmberg C, Heikinheimo M, Tryggvason K, Jalanko H: Expression of nephrin in pediatric kidney diseases. J Am Soc Nephrol 2001;12: 289-296.

$\checkmark 9$ Saleem MA, Ni L, Witherden I, Tryggvason K, Ruotsalainen V, Mundel P, Mathieson PW: Co-localization of nephrin, podocin, and the actin cytoskeleton: evidence for a role in podocyte foot process formation. Am J Pathol 2002; 161:1459-1466.

10 Shalhoub RJ: Pathogenesis of lipoid nephrosis: a disorder of T-cell function. Lancet 1974;2: 556-560.
11 Hariharan S, Adams MB, Brennan DC, Davis CL, First MR, Johnson CP, Ouseph R, Peddi VR, Pelz CJ, Roza AM, Vincenti F, George V: Recurrent and de novo glomerular disease after renal transplantation: a report from Renal Allograft Disease Registry (RADR). Transplantation 1999:68:635-641.

12 Le Berre L, Godfrin Y, Lafond-Puyet L, Perretto S, Le Carrer D, Bouhours JF, Soulillou JP, Dantal J: Effect of plasma fractions from patients with focal and segmental glomerulosclerosis on rat proteinuria. Kidney Int 2000; 58:2502-2511.

13 Savin VJ, Sharma R, Sharma M, McCarthy ET, Swan SK, Ellis E, Lovell H, Warady B, Gunwar S, Chonko AM, Artero M, Vincenti F: Circulating factor associated with increased glomerular permeability to albumin in recurrent focal segmental glomerulosclerosis. N Engl J Med 1996;334:878-883.

14 Sharma M, Sharma R, McCarthy ET, Savin VJ: 'The FSGS factor:' enrichment and in vivo effect of activity from focal segmental glomerulosclerosis plasma. J Am Soc Nephrol 1999; 10:552-561.

15 Sharma M, Sharma R, McCarthy ET, Savin VJ: The focal segmental glomerulosclerosis permeability factor: biochemical characteristics and biological effects. Exp Biol Med (Maywood) 2004;229:85-98.

16 Glassock RJ: Circulating permeability factors in the nephrotic syndrome: a fresh look at an old problem. J Am Soc Nephrol 2003; 14:541543.

17 Cattran D, Neogi T, Sharma R, McCarthy ET, Savin VJ: Serial estimates of serum permeability activity and clinical correlates in patients with native kidney focal segmental glomerulosclerosis. J Am Soc Nephrol 2003;14:448453.

18 Franz S FC, Mundel P: Cyclosporin protects podocyte stress fibers through stabilisation of synaptopodin protein expression. J Am Soc Nephrol 2005;16:109A.

19 Sharma R, Sharma M, McCarthy ET, Ge XL, Savin VJ: Components of normal serum block the focal segmental glomerulosclerosis factor activity in vitro. Kidney Int 2000;58:1973-1979.
20 Carraro M, Caridi G, Bruschi M, Artero M, Bertelli R, Zennaro C, Musante L, Candiano G, Perfumo F, Ghiggeri GM: Serum glomerular permeability activity in patients with podocin mutations (NPHS2) and steroid-resistant nephrotic syndrome. J Am Soc Nephrol 2002; 13:1946-1952.

21 Saleem MA, O'Hare MJ, Reiser J, Coward RJ, Inward CD, Farren T, Xing CY, Ni L, Mathieson PW, Mundel P: A conditionally immortalized human podocyte cell line demonstrating nephrin and podocin expression. J Am Soc Nephrol 2002;13:630-638.

22 Coward RJ, Foster RR, Patton D, Ni L, Lennon R, Bates DO, Harper SJ, Mathieson PW, Saleem MA: Nephrotic plasma alters slit diaphragm-dependent signaling and translocates nephrin, podocin, and CD2 associated protein in cultured human podocytes. J Am Soc Nephrol 2005;16:629-637.

23 Trouet D LR, Ni L, Mathieson PW, Saleem MA: Downregulation of TRPC6 by intact nephrin in the presence of human plasma - a protective mechanism for the maintenance of podocyte foot process structure. J Am Soc Nephrol 2005; 16:433A.

24 Huber TB, Hartleben B, Kim J, Schmidts M, Schermer B, Keil A, Egger L, Lecha RL, Borner C, Pavenstadt H, Shaw AS, Walz G, Benzing T: Nephrin and CD2AP associate with phosphoinositide 3-OH kinase and stimulate AKTdependent signaling. Mol Cell Biol 2003;23: 4917-4928.

25 Foster RR, Hole R, Anderson K, Satchell SC, Coward RJ, Mathieson PW, Gillatt DA, Saleem MA, Bates DO, Harper SJ: Functional evidence that vascular endothelial growth factor may act as an autocrine factor on human podocytes. Am J Physiol Renal Physiol 2003; 284:F1263-F1273.

26 Satchell SC, Harper SJ, Tooke JE, Kerjaschki D, Saleem MA, Mathieson PW: Human podocytes express angiopoietin 1, a potential regulator of glomerular vascular endothelial growth factor. J Am Soc Nephrol 2002;13:544-550. 